\title{
Atomization and Droplet Dynamics Analysis of Air-assisted Spray under Different Gas-liquid Ratios
}

\author{
Hao $\mathrm{Wu}^{1}$, Zhenyu Zhang ${ }^{* 1}$, Xiao Wei ${ }^{1}$, Fujun Zhang ${ }^{1,2}$ \\ 1 School of Mechanical Engineering, Beijing Institute of Technology, Beijing, China \\ ${ }^{2}$ Shenzhen Research Institute, Beijing Institute of Technology, Shenzhen, China \\ ${ }^{*}$ Corresponding author email: zhenyu.zhang@bit.edu.cn
}

\begin{abstract}
The principal objective of the present work is to experimentally investigate the droplet behavior of air-assisted spray. Simultaneous measurements of droplet sizes and velocities were made by phase-Doppler particle analyzer with particular emphasis on droplet size distribution, gas-droplet interactions and droplet collision. The experimental results show that air-assisted spray is composed of bulk of tiny droplets entrained by high-speed air flow. The calculated Stokes numbers of selected tracer droplets with diameter less than $5 \mu \mathrm{m}$ show that these droplets follow the air flow faithfully and thus can be used to estimate the local air flow velocity. The Reynolds number of local gas phase was found to be larger than the order of $10^{4}$. The analysis of the disintegration of discrete droplets indicates that the sampled droplets basically will not undergo shear breakup, but there is a possibility of turbulent breakup (Lasheras et al. J. Fluid Mech., vol. 357, 1998, pp. 351-379). A simplified calculation method based on classical concepts of binary droplet collision were adopted to describe the collision of unequal-size droplets and collision outcome within spray under different gas to liquid ratios (GLR). The coalescence possibility of all the collision outcomes is relatively considerable and found to be influenced by GLR of air-assisted spray.
\end{abstract}

\section{Keywords}

Two-phase flow, atomization, droplet size distribution, droplet collision, GLR

\section{Introduction}

Numerous implementations of spray process can be found in various combustion applications, such as vehicle engines, gas turbines, rocket engines and industrial furnaces [1]. The well-atomized spray with homogeneous and comparatively smaller droplets helps to increase the specific surface area of liquid with ambient air, thereby promoting heat transfer and evaporation [2].

As a dominant atomization method, the air-assisted atomization uses an additional medium (high velocity air stream) to enhance liquid disintegration [3]. The principle of airassisted atomization is based on the high shear stresses that develop at the interface between liquid-phase and gas-phase [4]. Depending on where gas-phase and liquid-phase come into contact, the air-assisted atomization can be categorized into internal-mixing and externalmixing types [2]. The internal-mixing air-assisted atomization can significantly improve atomization under low pressure and gas consumption. Since the impact between the highvelocity atomizing air and liquid takes place within the atomizer body, the design for internalmixing air-assisted atomizer provides great flexibility in internal configurations, as indicated in Ref $[5,6]$. In addition, the gas-liquid ratio, internal flow condition and orifice structure will exert various influences on the way the liquid disintegrates and how the spray develops over time and space. Hence, the atomization process of internal-mixing air-assisted spray normally involves various mechanisms which may interact with each other, such as Kelvin-Helmholtz instabilities and Rayleigh-Taylor instabilities [7]. For air-assisted atomizer, droplet diameter 
inversely depends on the gas to liquid mass flow ratio (GLR). For a given geometry, droplet diameter can be reduced by increasing gas speed, and/or decreasing the liquid flow rate. Experimental investigations have confirmed the hypothesis of transition from a bubble flow (low GLR) to a plug flow and further to the annular flow (high GLR) [8]. At low GLR, the breakup is based on the gas bubble explosions. When a gas bubble passes through the exit orifice, it expands in both radial and axial direction. Then the liquid film enveloping the gas bubble is stretched out hence causes film thinning. As the GLR increases, the bubbles become larger thus the thickness of the liquid film is reduced.

Moreover, a considerable number of the research work with respect to droplet dynamics and collision-coalescence mechanism have covered the revealing of the basic physical nature of binary droplet collision [9-11] and its influences on spray flow in actual applications [12]. Contrary to atomization, droplet collision is more likely to cause droplet coalescence, which will increase the size of individual droplet. This phenomenon is quite common within the dense spray region downstream of the atomizer. In spray application, it is relatively difficult to accurately describe the collision between the small droplets produced within the whole spray flow field because the number of droplets in the spray is so large that the collision between each other cannot be determined since the collision might occur between droplets of various sizes, with a variety of collision parameters, at various spray positions and in multiple droplets successively colliding. Therefore, the model simplification study of the droplet collision of the spray may not reflect the real spray droplet colliding dynamics, but it can be considered as statistically close to the real situation. The present paper deals with an internal mixing airassisted atomizer with an annular exit. Our aim is to examine its atomization characteristics, gas-liquid interaction and the dynamics of spray droplets.

\section{Experimental Setup}

The experimental injection system is composed of a plain liquid injector and an airassisted twin-fluid atomizer. The atomizing air is provided with a stabilized pressure of 700 $\mathrm{kPa}$ while the liquid pressure is stabilized at $800 \mathrm{kPa}$. The test liquid of this study is $\mathrm{n}$-dodecane. Figure 1 shows the section view of the air-assisted atomizer. The head of nozzle shell is designed with a hemispherical deletion, which can match well with the needle valve tip of a hemispherical entity. When the nozzle is closed, the hemispherical needle valve tip is tightly attached to the spherical surface of the shell head under the action of the spring force to achieve sealing. When the nozzle is open, an annular exit with diverging section which is analogous to the shape of Laval nozzle is formed between the surface of hemispherical tip of the needle valve and the spherical surface of the shell head. In this study, GLR is varied by altering the mass flow of injected liquid under constant air injection mass flow $(3.54 \mathrm{E}-03 \mathrm{~kg} / \mathrm{s})$.

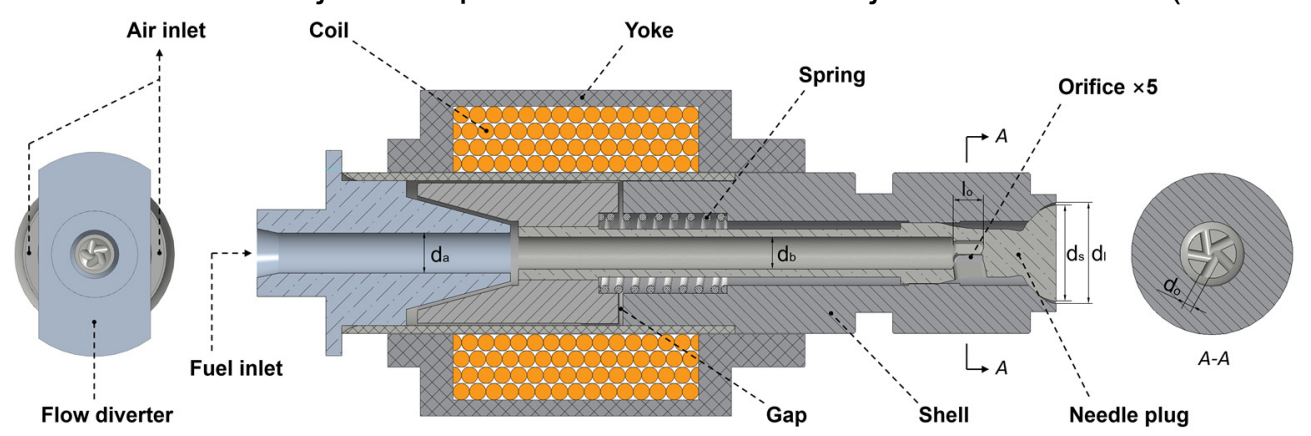

Figure 1. Section view of the internal-mixing air-assisted atomizer with detail view of flow diverter and swirl chamber. $\left(d_{a}=3.5 \mathrm{~mm}, \mathrm{~d}_{b}=3.0 \mathrm{~mm}, \mathrm{~d}_{\mathrm{s}}=6.0 \mathrm{~mm}, \mathrm{~d}_{\mathrm{l}}=6.6 \mathrm{~mm}\right)$ 
The simultaneous measurements of spray droplet size and velocity were conducted with a 2-D phase-Doppler particle analyser (PDPA), see Figure 2.

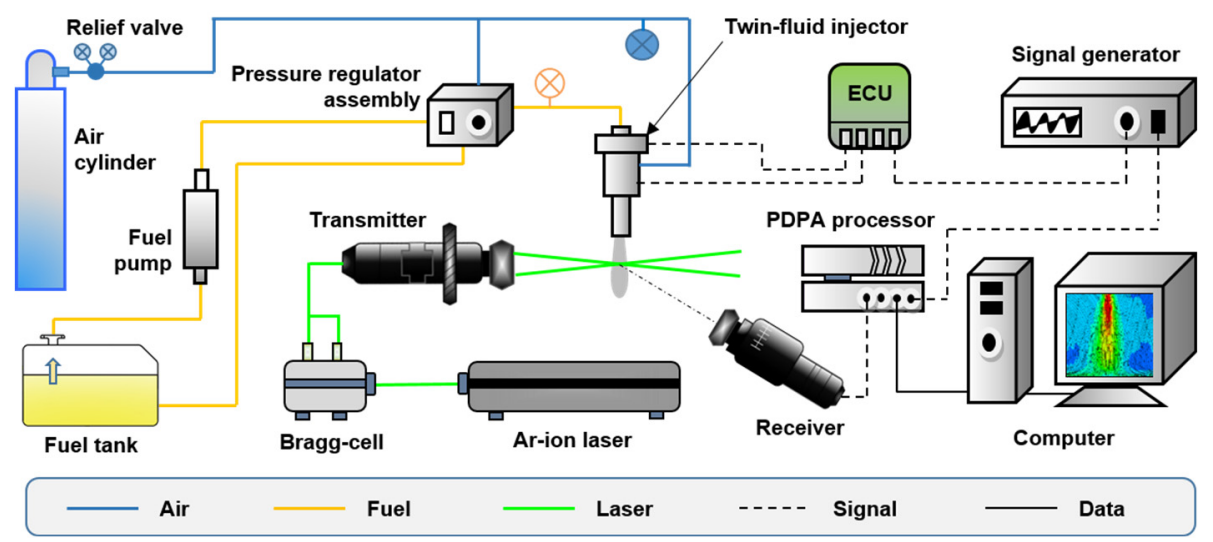

Figure 2. Schematic diagram of experimental setup for PDPA measurement.

The PDPA system principally consists of stand-alone Ar-ion laser, Bragg cell, transmitter, receiver, and signal processor hardware and software. In this experiment, a multiline Ar-ion laser produced a horizontally polarized light beam ( $0.8 \mathrm{~W}$ output power), which was split into four beams including two green $(\lambda=514.5 \mathrm{~nm})$ and two blue $(\lambda=488 \mathrm{~nm})$. The laser beam with the wavelength of $514.5 \mathrm{~nm}$ is used to measure the droplet axial velocity and diameter while the laser beam with the wavelength of $488 \mathrm{~nm}$ is used to measure the radial or tangential velocity of the droplet. The receiver probe was positioned at an angle of $70^{\circ}$ from the forward direction to collect the light scattered from droplets and to minimize reflections. The PDPA measurement volume was located $50 \mathrm{~mm}$ downwards along the spray centerline. A selfdeveloped ECU was used to simultaneously output the drive signal of the injection system and the trigger signal for PDPA. To avoid the influence of the last cyclic spray, the frequency of injection should not exceed $0.1 \mathrm{~Hz}$.

\section{Results and Discussion}

For air-assisted spray, accurate description of droplet size distribution is essential for further analysis, such as phase change, heat transfer, evaporation rate and the quality of combustible mixture formation. In addition, droplet size plays an important role in governing the two-phase flow and the dynamics of the droplets themselves. The distribution characteristics of droplet size can generally be defined as monodisperse and polydisperse [13]. A monodisperse distribution is one in which droplet sizes are mostly close to a single size level and such spray is usually described as quite uniform. By contrast, a polydisperse distribution suggests a non-uniform characteristic which presents a wide range of droplet sizes. Here, the probability density function (PDF) of discrete droplet diameter from three GLR cases were shown in Figure 3. The PDF was profiled with histogram as a function of droplet diameter $D_{i}$ with the interval of $\Delta D=2.0 \mu \mathrm{m}$.
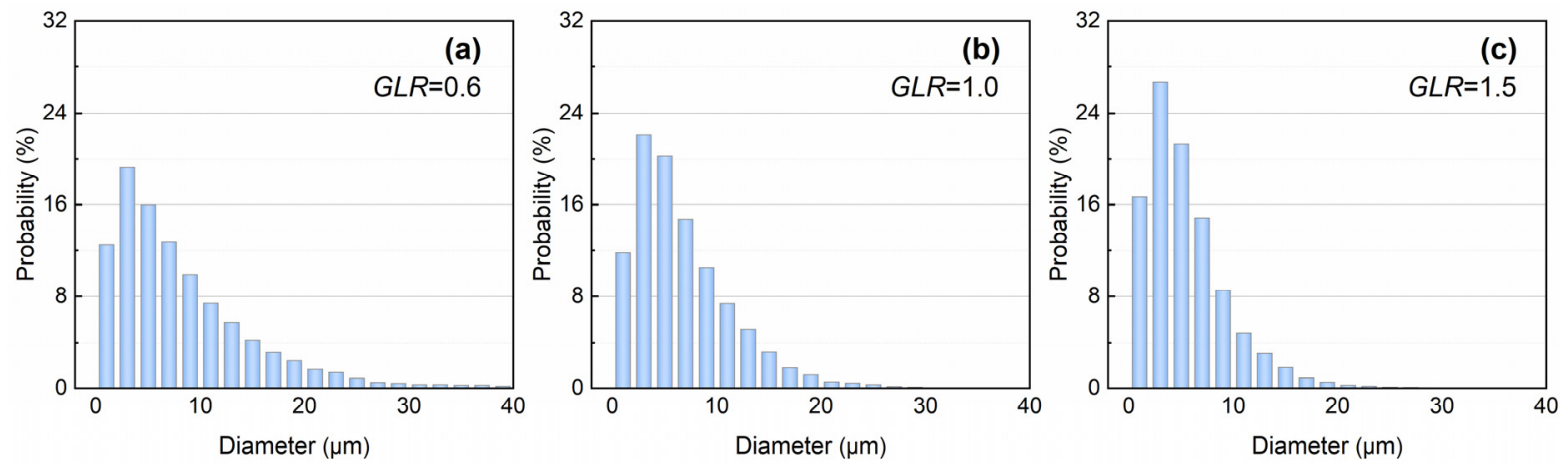
Figure 3. Probability of droplet size distribution for different gas-liquid mass ration cases: (a) GLR=0.6, (b) GLR=1.0, (c) GLR=1.5.

One can see that the sampling droplets under different GLR cases mostly fall into the range of $0-10 \mu \mathrm{m}$ in diameter, which indicates a relatively ideal atomization results for this spray. As GLR increases from 0.6 to 1.5 , the distribution probability of small droplets (less than $10 \mu \mathrm{m}$ ) gradually increases from $70 \%$ to $88 \%$. This result preliminarily shows that within a certain range, the increase of the gas-liquid mass ratio helps to improve the atomization quality of air-assisted spray. In addition, the peak value of droplet distribution that appears between 0-10 $\mu \mathrm{m}$ increases as GLR increases thus presents more notable polydisperse characteristic.

The mean droplet diameter and velocity are shown in Figure 4. Here both Sauter Mean Diameter (defined by $D_{32}$ ) and arithmetic mean diameter (defined by $D_{10}$ ) are used to characterize droplet mean diameter. Besides, droplet mass-averaged velocity $V_{m}$ and arithmetic mean velocity $V_{10}$ are used to manifest sampling droplet mean velocity. It can be found that droplet mean diameter shows a decreasing function of GLR while droplet mean velocity increases with the increasing of GLR. Therefore, it can be considered that the increase of GLR will increase the velocity of droplet movement and reduce the droplet size.

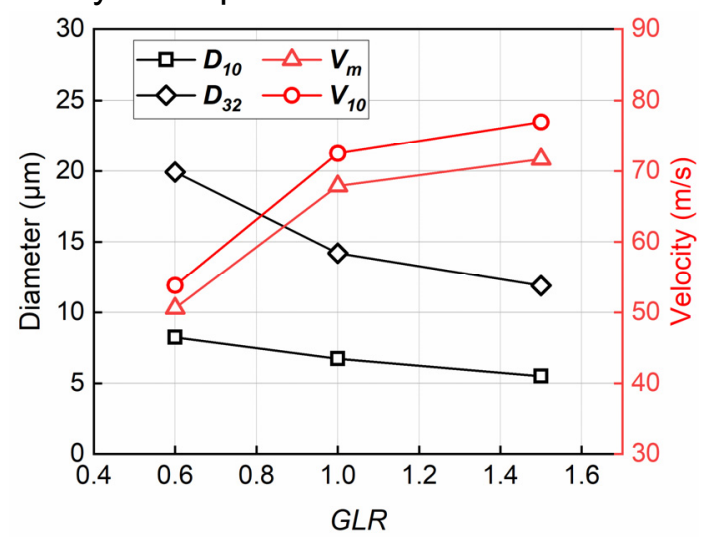

Figure 4. Mean droplet diameter and velocity.

Due to the unique gas-liquid twin-fluid characteristics of air-assisted spray, it is necessary to further characterize the basic flow behavior of the spray field and analyze the gas-liquid interaction. For air-assisted spray, tiny droplet motion is normally dominated by the degree to which droplet follows the airflow, which can be characterized by a dimensionless number of Strokes number (St). A droplet with a low St tends to follow fluid streamlines and therefore present perfect advection, while a droplet with a large St is dominated by its inertia and continues along its original trajectory [14].

According to the Ref [15], droplet motion in turbulent flow is directly related to the droplet's ability to respond to the large turbulent structure of the spray jet. As a result, Ferrand and Bazile et al. [16] defined the droplet St as the ratio of corresponding aerodynamic time constant $\left(\tau_{d}\right)$ to an appropriate turbulent time scale $\left(\tau_{L}\right)$, as $S t=\tau_{d} / \tau_{L}$. The corresponding aerodynamic time constant of droplet is defined as $\tau_{d}=\rho_{l} D^{2} / 18 \mu_{g}$, where $D$ is the droplet diameter, $\rho_{\mathrm{l}}$ and $\mu_{\mathrm{g}}$ represent the density of liquid droplet and dynamic viscosity of air, respectively. $\tau_{L}$ is chosen as the ratio of a large eddy length scale $R_{1 / 2}$ (the half width of the spray jet, selected from mean velocity profile and for a conservative estimate here) to the root mean square (RMS) of the centerline axial velocity fluctuations of airflow [15]. The droplets with the diameter of 0-5 $\mu \mathrm{m}$ were selected as "tracer droplets" and temporarily assumed to follow the airflow almost completely to ascertain instantaneous local airflow velocity. Thus, the RMS of velocity fluctuations can be calculated as 


$$
\mathrm{U}_{\mathrm{rms}}=\left[\frac{1}{\mathrm{n}} \times \sum_{\mathrm{i}=1}^{\mathrm{n}}\left(\mathrm{U}_{\mathrm{i}}-\overline{\mathrm{U}}\right)^{2}\right]^{\frac{1}{2}}
$$

where $\bar{U}$ is the arithmetic mean of axial velocity from $n$ droplets with diameter less than $5 \mu \mathrm{m}$, and $U_{i}$ is the axial velocity of the $i^{\text {th }}$ droplet. Therefore, the St of each sampled droplet of different GLR cases is calculated and presented in Figure 5, in which the grey region represents the location of tracer droplets. It can be found that the St of the tracer droplets does not exceed 0.1 for all GLR cases. Therefore, it can be confirmed that the tracking accuracy error of adopting droplets with diameter of $0-5 \mu \mathrm{m}$ to characterize the local airflow velocity is less than $1 \%[13,14]$. Moreover, this method also verifies the feasibility of using tiny droplets to characterize local air velocity, whereby the local airflow velocity can be calculated by $\mathrm{U}_{\mathrm{g}}=\sqrt{\left\langle\mathrm{U}_{\mathrm{i}} \mathrm{U}_{\mathrm{i}}\right\rangle}$. The calculated local airflow velocity of different GLR cases are listed in Table 1.
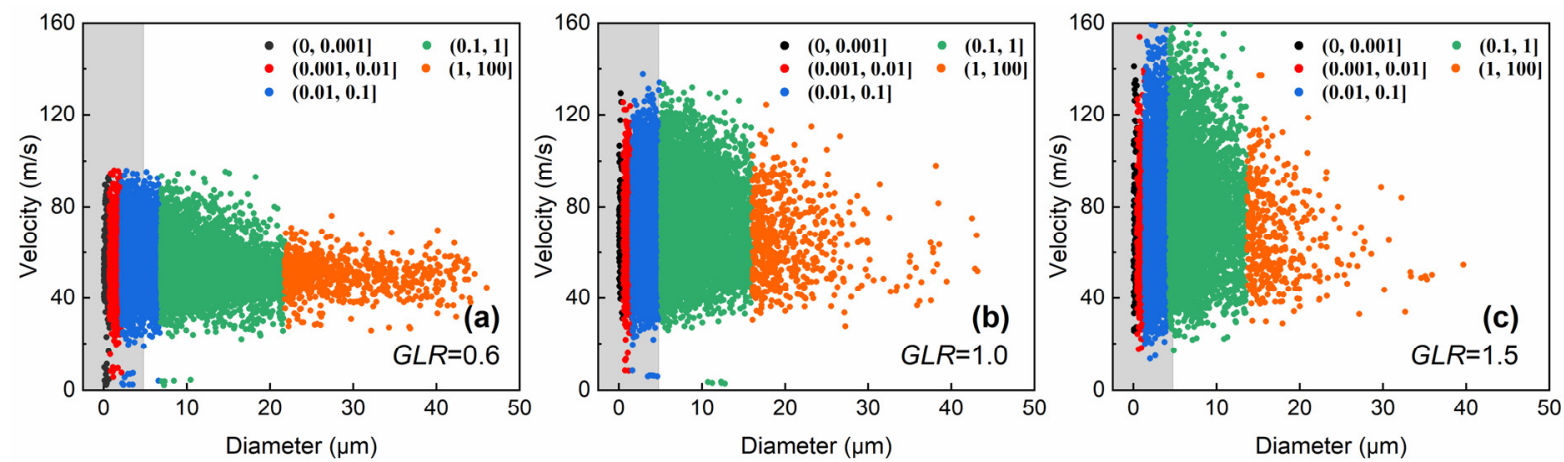

Figure 5. Range of droplet Stokes number for different gas-liquid mass ration cases: (a) $G L R=0.6$, (b) $G L R=1.0$, (c) $G L R=1.5$.

The Reynolds number $\left(\mathrm{Re}_{\mathrm{g}}\right)$ of the local airflow field can be calculated based on the obtained local airflow velocity $U_{g}$ and half-width of the jet spray (large eddy length scale $R_{1 / 2}$ ), given by $R_{g}=\rho_{g} U_{g} R_{1 / 2} / \mu_{g}$ [15]. The calculated $R_{g}$ of the local airflow of various GLR cases is shown in Table 1. The calculated $\mathrm{Re}_{\mathrm{g}}$ is basically within the order of $10^{4}-10^{5}$, whereby it can be concluded that the airflow field possesses high-intensity turbulence characteristics.

Table 1 - Turbulence characteristics of air-flow in spray field

\begin{tabular}{cccc}
\hline GLR & 0.6 & 1.0 & 1.5 \\
\hline $\mathrm{R}_{1 / 2}(\mathrm{~mm})$ & 13.80 & 12.50 & 12.00 \\
$\mathrm{U}_{\mathrm{rms}}(\mathrm{m} / \mathrm{s})$ & 11.91 & 19.85 & 26.18 \\
$\tau_{L}(\mathrm{~ms})$ & 1.16 & 0.63 & 0.46 \\
$\mathrm{U}_{\mathrm{g}}(\mathrm{m} / \mathrm{s})$ & 56.19 & 74.28 & 80.53 \\
$\mathrm{Re}_{\mathrm{g}}$ & 55701 & 66697 & 69416 \\
$\mathrm{St}\left(D_{10}\right)$ & 0.143 & 0.176 & 0.161 \\
$\mathrm{St}(d=5 \mu \mathrm{m})$ & 0.05 & 0.08 & 0.10 \\
\hline
\end{tabular}

When discrete droplets are exposed to high turbulence airflow, the force acting on a droplet can be regarded as the sum of a force generated by the relative velocity (also called slip velocity) between droplet and surrounding airflow, and a force caused by the turbulence of the carrier flow. Hence, the droplet breakup process caused by these two different forces is usually distinguished into "shear break-up" and "turbulent break-up" [17]. The classical theory believes that droplet break-up occurs when the shear Weber number $\left(\mathrm{We}_{\mathrm{s}}\right)$ of droplet which is exposed to a surrounding airflow exceeds a critical value

$$
\mathrm{We}_{\mathrm{s}}=\frac{\rho_{\mathrm{g}}\left(\mathrm{U}_{\mathrm{i}}-\mathrm{U}_{\mathrm{g}}\right)^{2} \mathrm{D}}{\sigma}>\left(\mathrm{We}_{\mathrm{s}}\right)_{\mathrm{c}}
$$


Hinze's [18] research on water droplets found that the $\left(\mathrm{We}_{\mathrm{s}}\right)_{\mathrm{c}}$ is of the order of 10 and this value will increase as the droplet Ohnesorge number (Oh) increases when considering liquid viscosity as $\left(\mathrm{We}_{\mathrm{s}}\right)_{\mathrm{c}}=\left(\mathrm{We}_{\mathrm{s}}\right)_{\mathrm{c} 0} \times[1+\mathrm{f}(\mathrm{Oh})]$. The velocity is plotted against diameter for all the sampled droplets to obtain the corresponding range of calculated $\mathrm{We}_{\mathrm{s}}$ of different GLR conditions, as shown in Figure 6. Obviously, the $\mathrm{We}_{\mathrm{s}}$ of all the sampled droplets is less than 10 , which confirms that there is no possibility for shear break-up of the droplets. In addition, Lasheras et al [17] pointed that when the gas-phase Reynolds number is of the order of 104$10^{5}$, the droplets within the turbulent airflow will undergo turbulent breakup. The above analysis shows that the Reynolds number of the gas-phase flow in the air-assisted spray field is large than 50,000 , so it can be inferred that the droplets in the spray may further disintegrate due to surrounding turbulent air flow.
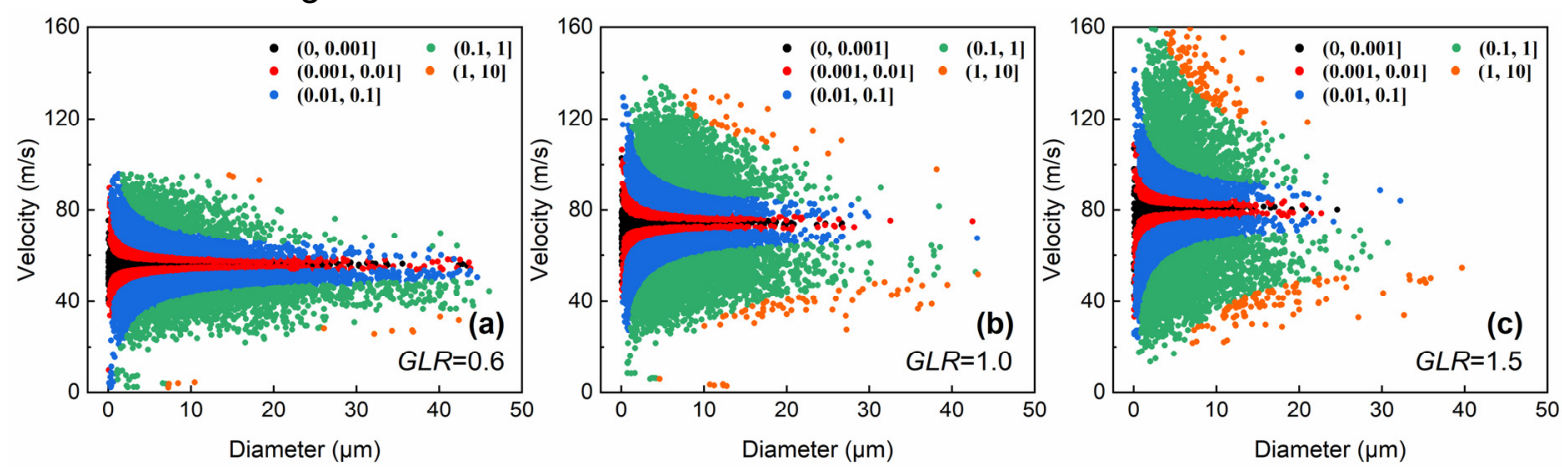

Figure 6. Range of droplet Weber number for different gas-liquid mass ration cases: (a) GLR=0.6, (b) GLR=1.0, (c) $G L R=1.5$.

Based on the simplified droplet collision description method proposed by Wu et al. [19], we conduct a comparative study on air-assisted spray droplet collisions under different GLR conditions. For a brief introduce here, it is assumed that the droplet collision only occurs in the form of head-on between two adjacent droplets captured in sequence when the velocity of the front droplet is lower than that of the droplet chasing behind. On the other hand, the collision will not occur when the velocity of the front droplet is greater than that of the rear droplet. Then, the outcome of droplet collision is based on the classic binary droplet collision regime diagram proposed by Qian and Law [10], and the influence of different droplet size ratios proposed by Tang et al. [11] on droplet collision is also considered. According to the three critical collision weber number $\mathrm{We}_{\mathrm{c}}=\rho_{\mathrm{l}} \mathrm{D}_{\mathrm{s}} \mathrm{U}_{\mathrm{r}}{ }^{2} / \sigma$, where $\mathrm{U}_{\mathrm{r}}$ and $\mathrm{D}_{\mathrm{s}}$ represent the relative velocity of the colliding droplet and the diameter of the small droplet, respectively. Then the colliding outcome can be divided into: coalescence (I), bouncing (II), coalescence (III) and separation (IV). The critical collision Weber number between the two adjacent collision results are $\mathrm{We}_{1}, \mathrm{We}_{2}$ and $\mathrm{We}_{3}$. According to the experimental results of Jiang et al. [9], We $\mathrm{e}_{1}$ can be estimated as 2.40, while $\mathrm{We}_{2}$ can be determined as 8.64 for $\mathrm{n}$-dodecane. In terms of $\mathrm{We}_{3}$, the droplet size ratio $\Delta$ that affects droplet coalescence has be taken into account based on the research of Tang et al. [11]. Therefore, statistical calculations on the distribution of collision outcomes of sampled droplets under different GLR conditions in the parameter space of $U_{r}$ and $D_{s}$, is shown in Figure 7. 

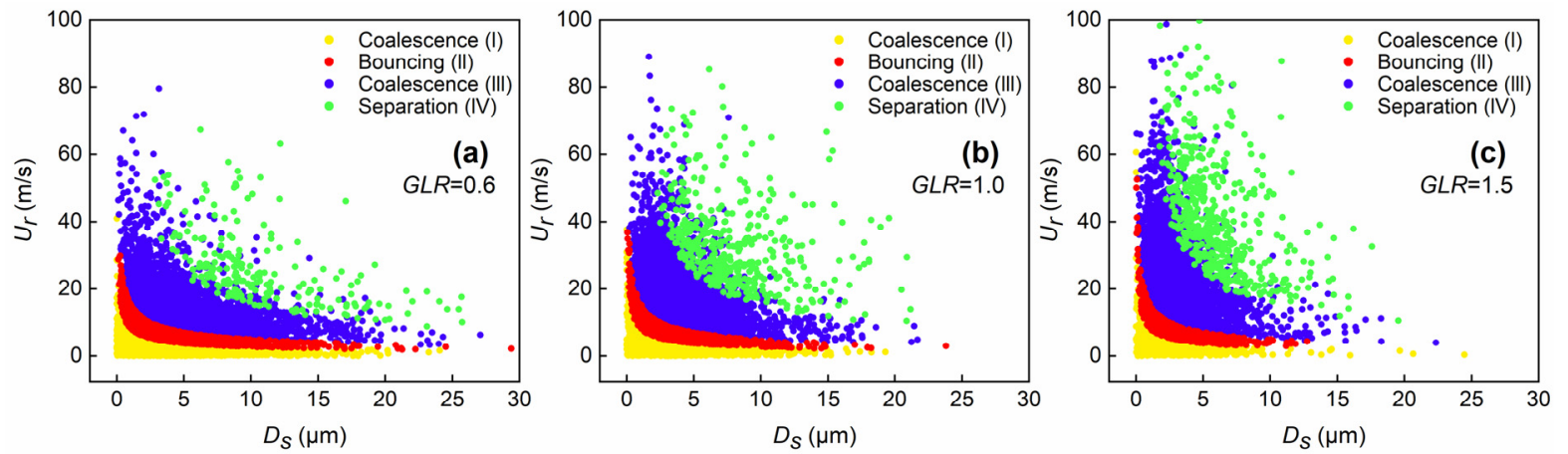

Figure 7. Droplet collision outcome distribution for different gas-liquid mass ration cases: (a) GLR=0.6, (b) $\mathrm{GLR}=1.0$, (c) $\mathrm{GLR}=1.5$.

The possible outcomes for each collision are marked with different colors. It is seen that all probable droplet collision outcomes can be observed for air-assisted spray. In addition, the relative velocity of the colliding droplets and the diameter of the small droplets are found to be more concentrated close to the zero point. This is because the two droplets sampled one after another will not differ much in velocity and size normally. Figure 8 shows the frequency of each type of collision outcome against various GLR obtained by further statistics. In light of the statistical results for all GLR cases, it can be found that the frequency of coalescence (III) with large deformation is the largest, followed by coalescence (I) and bouncing (II). The possibility of separation (IV) after coalescence occurs is lowest. With the increasing of GLR, the possibility of coalescence (III) and separation (IV) shows a monotonically increasing tendency while the other outcomes all decreases monotonically. This result preliminarily indicates that the increase of GLR will promote the coalescence after substantial deformation of colliding droplets in the air-assisted spray.

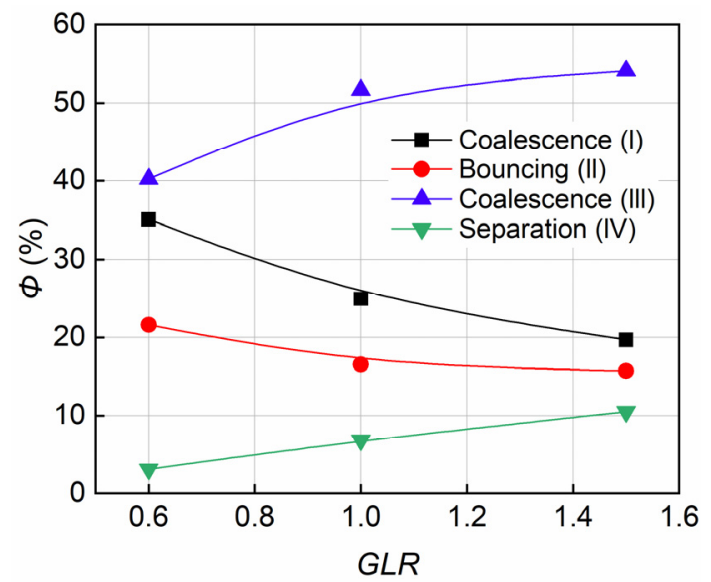

Figure 8. Probability of various droplet collision outcomes under different GLR conditions.

The explanation of the phenomenological description of the above calculation results is mainly based on the consideration of droplet velocity and size distribution range under different GLR conditions. In this study, when the sampled droplet data is sufficiently large, the statistical calculation results generally displays the overall characteristics of data which will not be affected by one single droplet. Therefore, it is considered that the droplet diameter of sampled droplets for calculating $\mathrm{We}_{\mathrm{c}}$ has negligible influence on the overall calculation result, which can be seen from the droplet diameter range shown in Figure 7. However, the $U_{r}$ therein will determine the actual value of $W_{c}$, which will increase with the calculate $U_{r}$ since higher GLR case tends to produce higher velocity droplets. Since the critical Weber number $\mathrm{We}_{1}$ and $\mathrm{We}_{2}$ are fixed, the obtained probability of coalescence (III) and separation (IV) consequently increases while the probability of coalescence (I) and bouncing (II) decreases. 


\section{Conclusions}

The atomization characteristics of air-assisted spray and droplet dynamics were investigated with phase-Doppler particle analyzer. The droplet diameter is predominantly distributed in the small-scale range of $0-10 \mu \mathrm{m}$ and the average velocity and diameter of the droplets increase and decrease respectively with the increase of GLR. The spray droplet with the diameter of 0-5 $\mu \mathrm{m}$ were selected as tracer droplet and the mean square velocity of these droplets was used to characterize the local gas-phase flow velocity. The Stokes number of these tracer droplet were calculated and the tracing accuracy errors are less than $1 \%$. The calculated Reynolds number of the gas-phase flow is in the order of $10^{4}-10^{5}$. It is inferred that there is possibility of turbulent breakup of the droplets. Based on the classic binary droplet collision theory and a simplified droplet collision model, it is inferred that the collisions between droplets that are sampled in sequence are most likely to occur. Therefore, on the premise of considering the effective relative velocities of sampled adjacent droplets and the droplet diameter ratio, the outcomes of all colliding droplets are calculated and distinguished according to the critical collision Weber numbers between various regimes.

\section{Acknowledgments}

The authors would like to acknowledge the research grant received from the National Natural Science Foundation of China (Grant No. 51806013) and the foundation research funds of Ministry of Industry and Information Technology (Grant No. JCKY2019602D018).

\section{References}

[1] Wu, H., Zhang, F., Zhang, Z., and Gao, H., 2020, "Experimental investigation on the spray characteristics of a self-pressurized hollow cone injector," Fuel, 272.

[2] Lefebvre, A. H., and McDonell, V. G., 2017, Atomization and sprays.

[3] Wu, H., Zhang, F., Zhang, Z., Guo, Z., Zhang, W., and Gao, H., 2020, "On the role of vortex-ring formation in influencing air-assisted spray characteristics of n-heptane," Fuel, 266.

[4] Gao, H., Zhang, F., Zhang, Z., Wang, S., and Wu, H., 2019, "Trajectory deviation of target jet of air-assisted spray under different conditions," Fuel, 249, pp. 252-263.

[5] Sovani, S. D., Sojka, P. E., and Lefebvre, A. H., 2001, "Effervescent atomization," Progress in Energy and Combustion Science, 27(4), pp. 483-521.

[6] García, J. A., Lozano, A., Alconchel, J., Calvo, E., Barreras, F., and Santolaya, J. L., 2017, "Atomization of glycerin with a twin-fluid swirl nozzle," International Journal of Multiphase Flow, 92, pp. 150-160.

[7] Qin, L. Z., Yi, R., and Yang, L. J., 2018, "Theoretical breakup model in the planar liquid sheets exposed to highspeed gas and droplet size prediction," International Journal of Multiphase Flow, 98, pp. 158-167.

[8] Ju, D., Fang, J., Zhang, T., Qiao, X., Xiao, J., and Huang, Z., 2015, "High-speed shadow imaging in internal flow pattern and macroscopic characteristics of a R134a flash-boiling spray discharged through a vertical twinorifice atomizer," International Journal of Multiphase Flow, 75, pp. 224-236.

[9] Jiang, Y. J., Umemura, A., and Law, C. K., 1992, "An experimental investigation on the collision behaviour of hydrocarbon droplets," Journal of Fluid Mechanics, 234, pp. 171-190.

[10] Qian, J., and Law, C. K., 1997, "Regimes of coalescence and separation in droplet collision," Journal of Fluid Mechanics, 331, pp. 59-80.

[11] Tang, C., Zhang, P., and Law, C. K., 2012, "Bouncing, coalescence, and separation in head-on collision of unequal-size droplets," Physics of Fluids, 24(2).

[12] Sommerfeld, M., and Pasternak, L., 2019, "Advances in modelling of binary droplet collision outcomes in Sprays: A review of available knowledge," International Journal of Multiphase Flow, 117, pp. 182-205.

[13] Wu, H., Zhang, F., and Zhang, Z., 2021, "Fundamental spray characteristics of air-assisted injection system using aviation kerosene," Fuel, 286.

[14] Tropea, C., Yarin, A. L., and Foss, J. F., 2007, Springer Handbook of Experimental Fluid Mechanics, Springer.

[15] Manish, M., and Sahu, S., 2019, "Droplet clustering and local spray unsteadiness in air-assisted sprays," Experimental Thermal and Fluid Science, 100, pp. 89-103.

[16] Ferrand, V., Bazile, R., Borée, J., and Charnay, G., 2003, "Gas-droplet turbulent velocity correlations and twophase interaction in an axisymmetric jet laden with partly responsive droplets," International Journal of Multiphase Flow, 29(2), pp. 195-217.

[17] Lasheras, J. C., Villermaux, E., and Hopfinger, E. J., 1998, "Break-up and atomization of a round water jet by a high-speed annular air jet," Journal of Fluid Mechanics, 357, pp. 351-379.

[18] Hinze, J. O., 1955, "Fundamentals of the hydrodynamic mechanism of splitting in dispersion processes," AIChE Journal, 1(3), pp. 289-295.

[19] Wu, H., Zhang, F., and Zhang, Z., 2021, "Droplet breakup and coalescence of an internal-mixing twin-fluid spray," Physics of Fluids, 33(1). 